\title{
Identification of Dish-Based Dietary Patterns for Breakfast, Lunch, and Dinner and Their Diet Quality in Japanese Adults
}

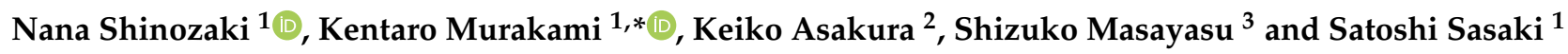 \\ 1 Department of Social and Preventive Epidemiology, School of Public Health, The University of Tokyo, \\ 7-3-1 Hongo, Bunkyo-ku, Tokyo 113-0033, Japan; nana-s@m.u-tokyo.ac.jp (N.S.); \\ stssasak@m.u-tokyo.ac.jp (S.S.) \\ 2 Department of Environmental and Occupational Health, School of Medicine, Toho University, \\ 5-21-16 Omori-Nishi, Ota-ku, Tokyo 143-8540, Japan; keiko.asakura@med.toho-u.ac.jp \\ 3 Ikurien-naka, 3799-6 Sugaya, Naka-shi, Ibaraki 311-0105, Japan; sizuko-masa@themis.ocn.ne.jp \\ * Correspondence: kenmrkm@m.u-tokyo.ac.jp; Tel.: +81-3-5841-7872
}

Citation: Shinozaki, N.; Murakami,

K.; Asakura, K.; Masayasu, S.; Sasaki, S. Identification of Dish-Based Dietary Patterns for Breakfast, Lunch, and Dinner and Their Diet Quality in Japanese Adults. Nutrients 2021, 13 , 67. https://doi.org/10.3390/ nu13010067

Received: 20 November 2020 Accepted: 21 December 2020 Published: 28 December 2020

Publisher's Note: MDPI stays neutral with regard to jurisdictional claims in published maps and institutional affiliations.

Copyright: () 2020 by the authors. Licensee MDPI, Basel, Switzerland. This article is an open access article distributed under the terms and conditions of the Creative Commons Attribution (CC BY) license (https: / / creativecommons.org/ licenses/by/4.0/).

\begin{abstract}
We identified dish-based dietary patterns for breakfast, lunch, and dinner and assessed the diet quality of each pattern. Dietary data were obtained from 392 Japanese adults aged 20-69 years in 2013, using a 4 d dietary record. K-means cluster analysis was conducted based on the amount of each dish group, separately for breakfasts $(n=1462)$, lunches $(n=1504)$, and dinners $(n=1500)$. The diet quality of each dietary pattern was assessed using the Healthy Eating Index 2015 (HEI-2015) and Nutrient-Rich Food Index 9.3 (NRF9.3). The extracted dietary patterns were as follows: 'bread-based' and 'rice-based' for breakfast; 'bread', 'rice-based', 'ramen', 'udon/soba', and 'sushi/rice bowl dishes' for lunch; and 'miscellaneous', 'meat dish and beer', and 'hot pot dishes' for dinner. For breakfast, the HEI-2015 and NRF9.3 total scores were higher in the 'rice-based' pattern than the 'bread-based' pattern. For lunch, the HEI-2015 and NRF9.3 total scores were relatively high in the 'rice-based' pattern and low in the 'ramen' pattern. For dinner, the HEI-2015 total score was the highest in the 'meat dish and beer' pattern, and the NRF9.3 total score was higher in the 'hot pot dishes' than the 'miscellaneous' pattern. These results suggested that breakfast, lunch, and dinner have distinctive dietary patterns with different diet qualities.
\end{abstract}

Keywords: mixed dish; dietary pattern; diet quality; meal; HEI-2015; NRF9.3; Japan

\section{Introduction}

Although many studies in nutritional epidemiology have focused on single foods and specific nutrients, this approach may be inappropriate in considering synergistic and interactive effects among foods and nutrients [1-3]. Therefore, a growing interest in dietary pattern analysis considers overall diet rather than individual foods or nutrients. Since dietary patterns may be easy for the public to translate into diets, dietary pattern analysis can be useful in developing public health implications [1]. Furthermore, because dietary patterns are characterised on the basis of eating behaviour, they can also facilitate understandings of dietary practice, and provide guidance for nutritional intervention and education [1].

Most work on dietary patterns has analysed overall dietary intake without differentiating eating occasions. However, since people eat foods in specific combinations at mealtimes [4-8], grouping breakfast, lunch, and dinner together may overlook the distinctive dietary patterns of each eating occasion [4]. To date, there have been several studies investigating meal-specific dietary patterns for breakfast $[9,10]$ and all main meals (breakfast, lunch, or dinner) $[4,11-13]$. These studies have indicated associations of specific dietary patterns at breakfast, lunch, or dinner with an overall dietary pattern [11], overall diet quality [9], and risk of hyperglycaemia [12]. 
Moreover, previous dietary pattern analyses have been conducted at the level of single foods or food groups, whereas people generally eat mixed dishes rather than single foods [14]. Mixed dishes reflect not only the quantities and combinations of individual foods, but also the varieties of elements related to eating behaviours, such as cooking practice, serving, and eating situations. Therefore, identification of dietary patterns at the level of mixed dishes, rather than single foods, would help us better understand human eating behaviours at meals. Furthermore, given that mixed dishes are the state of foods consumed, assessing dish-based dietary patterns at different eating occasions in relation to diet quality may be useful to develop practical dietary recommendations that people can easily understand.

However, to our knowledge, no study has investigated meal-specific dietary patterns based on mixed dishes. Therefore, the aim of the present study was to identify dish-based dietary patterns for breakfast, lunch, and dinner, and to assess the diet quality of each dietary pattern.

\section{Materials and Methods}

\subsection{Data Source}

This cross-sectional study was based on data from a nationwide dietary survey conducted in 20 study areas consisting of 23 prefectures in Japan between February and March 2013. Details of the survey have been described elsewhere [15,16]. In brief, 199 research dietitians working at separate welfare facilities recruited their colleagues and the family members of the colleagues as study participants. The recruitment was conducted such that each study area included approximately four apparently healthy subjects (two men and two women) from each of five 10-year age categories (20-29, 30-39, 40-49, 50-59, and 60-69 years). One individual per household could participate in the survey. None of the participants was a dietitian or a medical professional, had received dietary therapy by a doctor or dietitian, had a history of educational hospitalisation for diabetes mellitus, or was a pregnant or lactating woman. In total, 196 men and 196 women aged 20-69 years provided dietary data for the analysis. Body weight (to the closest $0.1 \mathrm{~kg}$ ) in light clothes and body height (to the closest $0.1 \mathrm{~cm}$ ) without shoes was measured using standardised procedures by research dietitians or medical workers. Body mass index (BMI) was calculated as body weight $(\mathrm{kg})$ divided by the square of body height $\left(\mathrm{m}^{2}\right)$.

\subsection{Dietary Assessment}

Dietary data were obtained using a 4-day weighed dietary record (DR), as described previously [16]. Briefly, participants were asked to record all foods and beverages consumed for four non-consecutive days (three working days and one non-working day, excluding days before and after a night shift). Research dietitians explained to the participants how to keep the DR and requested them to weigh foods and beverages with a provided digital scale or measuring spoon and cup. The main recorded items were dish names, food names (including beverages and any ingredients in dishes), and approximate amounts or measured weights of foods consumed. The participants submitted the recording sheets to a research dietitian at each facility shortly after recording. The research dietitians reviewed the recording sheets as soon as possible, and if necessary, asked participants additional information to clarify the name or amount of food on the sheet. The research dietitian at each facility coded each food item recorded in the column 'food names' using the Standard Tables of Food Composition in Japan [17] in a uniform procedure. All food codes and weights were then reconfirmed by two other research dietitians at the central office of the study.

\subsection{Definition of Breakfast, Lunch, and Dinner}

The DR sheet consisted of four sections (breakfast, lunch, dinner, and snacks). The identification of eating occasions was based on this classification; thus, a meal recorded in the sections of breakfast, lunch, or dinner on the recording sheet was considered the respective meal. Eating occasions including only water or dietary supplements were 
excluded in this study. In total, the number of meals was 1474 for breakfast, 1515 for lunch, and 1551 for dinner.

\subsection{Identification of Dish Groups}

To classify dish items consumed at each eating occasion into dish groups, each unique dish item in the column 'dish items' was coded using a recently developed dish composition database $[18,19]$. A detailed description of the database has been reported elsewhere [18,19]. Briefly, the dish composition database was developed based on a 16-day weighed DR completed by 252 Japanese adults. For a total of 371 dish groups, the database consisted of information on dish names, dish codes, portion size, and food groups and nutrient contents.

In this study, a total of 20,057 dishes (5492 for breakfast, 7263 for lunch, and 7302 for dinner) appeared in the DR. We coded each dish item using dish codes in the dish composition database, and consequently, all dishes were classified into 335 dish groups. To remove the complexity, these dish groups were further consolidated into 59 dish groups for the present analysis, based on similarity of dish name, ingredients, cooking methods, and energy and nutrient content (Supplementary Table S1). Refined grain dishes and wholegrain dishes were not separated in the process of grouping dishes, since wholegrain intake is very low in Japan $[8,11]$. After this process, the number of dish groups included in breakfast, lunch, and dinner was 54,59, and 59, respectively.

\subsection{Calculation of Diet Quality Scores}

Estimates of energy and nutrient content for individual meals were calculated based on the weight of food items and their nutrient content, using the Standard Tables of Food Composition in Japan [17]. For food items with unavailable data on the added sugar content in that database, added sugar values were calculated based on the same or similar food in the 2011-2012 Food Patterns Equivalents Database [20]. Teaspoon equivalents in the Food Patterns Equivalents Database were converted into grams by multiplying by 4.2 (grams of added sugar per teaspoon).

The diet quality of individual meals was assessed using the Healthy Eating Index 2015 (HEI-2015) [21,22] and Nutrient-Rich Food Index 9.3 (NRF9.3) [23,24]. These indices have been used to assess not only overall diet quality [25], but also individual meals [8,26-29]. The usefulness of HEI-2015 and NRF9.3 has been demonstrated in the Japanese population [30]. The HEI-2015 is a 100-point scale designed to assess the conformance of a set of foods with the 2015-2020 Dietary Guidelines for Americans [22,31], with a higher score indicating a better quality of diet. The nine adequacy components (maximum score) include total fruits (5), whole fruits (5), total vegetables (5), greens and beans (5), wholegrains (5), dairy (10), total protein (5), seafood and plant protein (5) and fatty acids (the ratio of the sum of polyunsaturated and monounsaturated fatty acids to saturated fatty acids) (10); the four moderation components include refined grains (10), sodium (10), added sugars (10) and saturated fats (10). We calculated the total and component scores of HEI2015 based on the energy-adjusted values in each meal (i.e., amount/4184 kJ of energy or percent of energy), except for fatty acids, using the 2011-2012 Food Patterns Equivalents Database [20].

The NRF9.3 is a composite measure of nutrient density, which can be applied to individual foods, meals, and menus or to the total diet [23,24]. The NRF9.3 is calculated as the sum of the percentage of reference daily values (RDVs) for nine qualifying nutrients minus the sum of the percentage of RDVs for three disqualifying nutrients. For qualifying nutrients, the RDV percentage of each was truncated at 100, so that a high intake of one nutrient could not compensate for a low intake of another. As such, the maximum possible score is 900 , with the higher NRF9.3 total score indicating a better quality of diet. RDVs were determined based on the Dietary Reference Intakes (DRIs) for Japanese, 2020 [32], except for added sugar (Supplementary Table S2). The Japanese DRIs present different values depending on sex and age categories. In this study, we used the RDVs for men aged 30-49 years to evaluate all meals uniformly. We used the Recommended Dietary 
Allowance for protein, vitamins A and C, calcium, iron, and magnesium, the tentative dietary goal for preventing lifestyle-related diseases for dietary fibre, potassium, saturated fats, and sodium, and the adequate intake for vitamin D. For added sugar, because the intake is low [33] and no recommended amount is shown in Japan, we used the conditional recommendation advocated by the World Health Organisation (i.e., upper limit of $5 \%$ of energy) [34]. For added sugar and saturated fats (RDVs are calculated as a percent of energy), the energy intake was determined from Estimated Energy Requirement for the moderate level of physical activity in the DRIs. We calculated the total and component scores of NRF9.3 based on nutrient content for individual meals, which was adjusted for the energy content of each meal by the density method.

\subsection{Data Analysis}

All analyses were conducted using the statistical software package SAS version 9.4 (SAS Institute Inc., Cary, NC, USA). For simplicity and ease of interpretation, dish groups representing less than $3 \%$ of the total number of meals at breakfast, lunch, and dinner were removed from the analysis. We calculated the consumption frequency of each dish group by dividing the number of dishes by the total number of meals separately for breakfast, lunch, and dinner. Cluster analysis was performed to divide individual meals into mutually exclusive groups based on the similarity of the amount of dish groups. We used the $k$-means method, which uses Euclidean distances between each meal to estimate clusters empirically from the data set, to avoid imposing a hierarchical structure on the clusters [35]. The input variables were the wet amount of dish groups (grams) in each meal. The variables were not standardised before entering into cluster analysis, because all the variables had the same unit (grams), and standardisation would dilute differences between clusters and reduce correlations between variables [35]. The FASTCLUS procedure in SAS was performed separately for breakfast, lunch, and dinner. As cluster analysis is sensitive to outliers, we removed them using the following two methods. First, we removed meals with a dish group whose amount was 5 standard deviations away from the mean of that dish group [36,37]. Second, we ran cluster analysis with a predefined number of 20 clusters for each meal, and removed meals in clusters with less than 8 meals [36]. Through these procedures, a total of 74 meals were removed, leaving the final samples of 1462, 1504, and 1500 meals for breakfast, lunch, and dinner, respectively.

The number of clusters had to be determined in the $k$-means method prior to analysis. To determine the most appropriate number of clusters, 2 to 8 cluster solutions were examined. The final cluster solution was selected based on a plot of the ratio of betweencluster variance to within-cluster variance vs. the number of clusters [38-40], a plot of within-cluster variance vs. the number of clusters [40], cubic clustering criterion [39,41], the sample size (cluster solutions were retained only if each cluster contained $5 \%$ of the total sample in each meal type) [42], and the interpretation of each cluster [41,43]. The reproducibility of the clusters was assessed separately for breakfast, lunch, and dinner, by performing the $k$-means cluster analysis repeatedly with the selected number of cluster solutions in random samples of $50 \%$ of the participants [43]. We confirmed that similar results were identified to those in the original analysis.

To describe the characteristics of each cluster, we calculated the means and standard errors of the amount of food groups, dish groups, and the total and component scores of HEI-2015 and NRF9.3 for each cluster in breakfast, lunch, and dinner. Food groups were based mainly on the Standard Tables of Food Composition in Japan [17] and the similarity of nutrient composition or culinary use of foods (Supplementary Table S3). Differences in the mean values of the amount of each food group and dish group, as well as the HEI-2015 and NRF9.3 total and component scores across clusters, were compared for each eating occasion using an independent t-test, or ANOVA followed by Tukey's test when appropriate. The clusters were basically labelled based on the dish groups with a significantly larger mean amount than any other clusters within each eating occasion. Two-sided $p$-values $<0.05$ were considered statistically significant. 


\section{Results}

Participants characteristics of the study participants have been provided elsewhere [16]. The present analysis included 1587 days of dietary data obtained from 392 Japanese adults with a mean age of 44.5 years (SD 13.4) and a mean BMI of $23.3 \mathrm{~kg} / \mathrm{m}^{2}$ (SD 3.6). Of the 59 dish groups, the numbers of dish groups consumed in $\geq 3 \%$ of the total number of meals were 24,37 , and 37 for breakfast, lunch, and dinner, respectively.

Table 1 shows the consumption frequency of dish groups with a consumption frequency of $3 \%$ or more in all breakfasts, and the weight $(\mathrm{g})$ of dish groups in each dietary pattern. The most frequently consumed dish groups were Japanese and Chinese tea, coffee/tea, rice, plain bread, and miso soup.

Table 1. Consumption frequency of dish groups in all breakfasts ${ }^{1}$ and weight $(\mathrm{g})$ of dish groups in each dietary pattern ${ }^{2}$.

\begin{tabular}{|c|c|c|c|c|c|c|c|c|}
\hline \multirow[b]{4}{*}{ ID } & \multirow{3}{*}{ Dish Group } & \multirow[b]{4}{*}{$n$} & \multirow{4}{*}{ 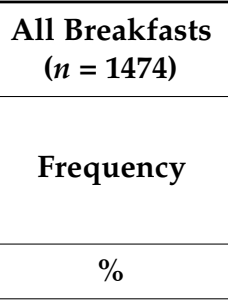 } & \multicolumn{5}{|c|}{$\begin{array}{l}\text { Breakfasts Included in Cluster Analysis }{ }^{3} \\
\qquad(n=1462)\end{array}$} \\
\hline & & & & \multicolumn{4}{|c|}{ Weight $(g)$} & \multirow{3}{*}{$p^{4}$} \\
\hline & & & & \multicolumn{2}{|c|}{$\begin{array}{l}\text { Bread-Based } \\
\quad(n=983)\end{array}$} & \multicolumn{2}{|c|}{$\begin{array}{l}\text { Rice-Based } \\
(n=479)\end{array}$} & \\
\hline & Name & & & Mean & SE & Mean & SE & \\
\hline 48 & Japanese and Chinese tea & 650 & 44.1 & 22.0 & 1.6 & 258.8 & 6.0 & $<0.0001$ \\
\hline 47 & Coffee $/$ tea & 602 & 40.8 & 118.5 & 4.0 & 22.7 & 2.9 & $<0.0001$ \\
\hline 1 & Rice & 474 & 32.2 & 27.8 & 2.1 & 99.1 & 4.3 & $<0.0001$ \\
\hline 8 & Plain bread & 424 & 28.8 & 31.2 & 1.4 & 10.5 & 1.4 & $<0.0001$ \\
\hline 55 & Miso soup & 365 & 24.8 & 25.7 & 2.3 & 113.6 & 5.9 & $<0.0001$ \\
\hline 27 & Fruit/fruit dishes & 305 & 20.7 & 20.4 & 1.5 & 17.3 & 1.8 & 0.22 \\
\hline 40 & Egg dishes & 260 & 17.6 & 10.2 & 1.0 & 18.2 & 1.7 & $<0.0001$ \\
\hline 42 & Yogurt/cheese & 242 & 16.4 & 15.7 & 1.3 & 17.6 & 2.1 & 0.40 \\
\hline 9 & Sweet bread/savoury bread & 231 & 15.7 & 21.2 & 1.6 & 10.2 & 1.7 & $<0.0001$ \\
\hline 41 & Milk/soymilk & 229 & 15.5 & 32.9 & 2.5 & 20.8 & 2.7 & 0.003 \\
\hline 22 & Raw or boiled vegetable dishes & 189 & 12.8 & 10.3 & 1.2 & 10.3 & 1.3 & 0.99 \\
\hline 49 & Cocoa/milk beverage & 146 & 9.9 & 17.5 & 1.9 & 14.2 & 2.2 & 0.29 \\
\hline 58 & Tsukudani/pickles & 144 & 9.8 & 1.2 & 0.2 & 4.9 & 0.6 & $<0.0001$ \\
\hline 17 & Natto (fermented soybeans) & 139 & 9.4 & 2.8 & 0.4 & 8.4 & 0.9 & $<0.0001$ \\
\hline 50 & Vegetable and fruit juice & 126 & 8.5 & 16.9 & 1.8 & 14.2 & 2.6 & 0.40 \\
\hline 39 & Processed meat dishes & 96 & 6.5 & 2.6 & 0.4 & 3.4 & 0.7 & 0.23 \\
\hline 30 & Grilled or stir-fried seafood dishes & 74 & 5.0 & 2.4 & 0.4 & 4.6 & 0.8 & 0.01 \\
\hline 6 & Rice ball & 72 & 4.9 & 6.6 & 1.1 & 13.5 & 2.6 & 0.005 \\
\hline 21 & Japanese-style vegetable side dishes & 70 & 4.7 & 1.4 & 0.3 & 4.9 & 1.0 & $<0.0001$ \\
\hline 56 & Western or Chinese soup & 68 & 4.6 & 11.4 & 1.6 & 5.2 & 1.5 & 0.02 \\
\hline 26 & Mushrooms/seaweed/konnyaku dishes & 52 & 3.5 & 0.1 & 0.1 & 1.6 & 0.5 & $<0.0001$ \\
\hline 44 & Ice cream/jelly/pudding/cake & 48 & 3.3 & 3.1 & 0.5 & 1.2 & 0.5 & 0.03 \\
\hline 16 & Potato dishes & 46 & 3.1 & 2.4 & 0.6 & 2.9 & 0.8 & 0.60 \\
\hline 33 & Canned fish/seafood delicacy & 44 & 3.0 & 0.3 & 0.1 & 0.8 & 0.2 & 0.009 \\
\hline
\end{tabular}

SE, standard error. ${ }^{1}$ The number of dishes per total number of breakfasts. In total, 5492 dishes (54 dish groups) were consumed at breakfast. Only 24 dish groups with a consumption frequency of $3 \%$ or more are shown. ${ }^{2}$ Each cluster represents a group of meals with a similar dish-based dietary pattern. ${ }^{3}$ Twelve meals were removed from cluster analysis; three meals with a dish group whose amount was five standard deviations away from the mean of that dish group, and nine meals that fell into clusters of less than eight meals when cluster analysis was conducted with a predefined number of 20 clusters. ${ }^{4}$ Differences between two dietary patterns were tested with an independent t-test.

The cluster analysis identified two dietary patterns. The predominant pattern $(n=983)$ was characterised by larger amounts of coffee/tea, plain bread, sweet bread/savoury bread, milk/soymilk, Western or Chinese soup, and ice cream/jelly/pudding/cake. Since there were many dish groups with a larger amount of consumption including bread, this pattern was named the 'bread-based' pattern. Meanwhile, the other pattern $(n=479)$, which was named the 'rice-based' pattern, was characterised by larger amounts of Japanese and Chinese tea, rice, miso soup, egg dishes, tsukudani/pickles, natto (fermented soybeans), grilled or stir-fried seafood 
dishes, rice ball, Japanese-style vegetable side dishes, mushrooms/seaweed/konnyaku dishes, and canned fish/seafood delicacy.

For lunch, the most frequently consumed dish groups were Japanese and Chinese tea, rice, raw or boiled vegetable dishes, miso soup, and fruit/fruit dishes (Table 2). Five clusters were identified. The 'bread' pattern $(n=781)$ was characterised by the largest amount of sweet bread/savoury bread and the smallest amount of Japanese and Chinese tea. The 'rice-based' pattern $(n=393)$ was characterised by the largest amounts of Japanese and Chinese tea, rice, egg dishes, and grilled or stir-fried meat dishes. Each of the other three clusters had one dish group with the largest amount: namely, the 'ramen' $(n=99)$, 'udon/soba' $(n=125)$, and 'sushi/rice bowl dishes' $(n=106)$ patterns.

For dinner, the most frequently consumed dish groups were Japanese and Chinese tea, rice, miso soup, raw or boiled vegetable dishes, and Japanese-style vegetable side dishes (Table 3). Three dietary patterns were identified. Most meals were categorised into the 'miscellaneous' pattern $(n=1211)$, which did not have any specific dish groups with a significantly larger amount than any other clusters. The 'meat dish and beer' pattern $(n=165)$ was characterised by the largest amounts of beer, gyoza dumplings/shumai/meatballs, and simmered meat with seasoning, and the smallest amounts of Japanese and Chinese tea and rice. The 'hot pot dishes' pattern $(n=124)$ was characterised by the largest amount of hot pot dishes, and the smallest amounts of miso soup, raw or boiled vegetable dishes, Japanese-style vegetable side dishes, grilled or stir-fried seafood dishes, grilled or stir-fried meat dishes, deep-fried meat dishes, and curry and rice/omelette rice.

The total and component scores of HEI-2015 and NRF9.3 for each dietary pattern are summarised in Table 4. For breakfast, the 'rice-based' pattern scored higher in the total score of HEI-2015 and its component scores for total vegetable, greens and beans, total protein foods, seafood and plant proteins, fatty acids, added sugars, and saturated fats. Meanwhile, the 'bread-based' pattern had higher HEI-2015 component scores for total fruits, whole fruits, dairy, refined grains, and sodium. For NRF9.3, the 'rice-based' pattern was higher in the total score and the component scores of protein, dietary fibre, vitamin A, vitamin $\mathrm{C}$, iron, and sodium, while the 'bread-based' pattern had higher component scores for added sugars and saturated fats.

For lunch, the HEI-2015 total score was higher in the 'rice-based' pattern than the 'bread', 'ramen', and 'udon/soba' patterns, and the lowest in the 'ramen' pattern. For the HEI-2015 component scores, the 'bread' pattern was the highest in dairy, and the 'udon/soba' was the highest in wholegrains. The NRF9.3 total score was higher in the 'bread', 'rice-based', and 'sushi/rice bowl dishes' patterns than the other two patterns. For the NRF 9.3 component scores, the 'rice-based' pattern was the highest in vitamin C, and the 'ramen' pattern was the highest in sodium. The 'sushi/rice bowl dishes' pattern had the lowest score for dietary fibre.

For dinner, the 'meat dish and beer' pattern was the highest in the HEI-2015 total score and the component scores for refined grains, sodium, and saturated fats. The 'miscellaneous' pattern was the lowest in the component score for refined grains. The 'hot pot dishes' pattern had the highest component scores for total vegetables and total protein foods, and the lowest component scores for fatty acids and saturated fats. The NRF9.3 total score was higher in the 'hot pot dishes' pattern than the 'miscellaneous' pattern. The 'meat dish and beer' pattern had the lowest component scores in many components (including protein, dietary fibre, vitamin A, calcium, iron, saturated fats, and sodium). In contrast, the 'hot pot dishes' pattern had the highest component scores in many components (including dietary fibre, vitamin C, calcium, iron, potassium, and saturated fats). The weight (g) of food groups for each dietary pattern is summarised in Supplementary Table S4. 
Table 2. Consumption frequency of dish groups in all lunches ${ }^{1}$ and weight $(\mathrm{g})$ of dish groups in each dietary pattern ${ }^{2}$.

\begin{tabular}{|c|c|c|c|c|c|c|c|c|c|c|c|c|c|c|}
\hline \multirow[b]{4}{*}{ ID } & \multirow{4}{*}{$\begin{array}{l}\text { Dish Group } \\
\text { Name }\end{array}$} & \multicolumn{2}{|c|}{$\begin{array}{l}\text { All Lunches } \\
(n=1515)\end{array}$} & \multicolumn{11}{|c|}{$\begin{array}{l}\text { Lunches Included in Cluster Analysis }{ }^{3} \\
\qquad(n=1504)\end{array}$} \\
\hline & & \multirow{2}{*}{\multicolumn{2}{|c|}{ Frequency }} & \multicolumn{10}{|c|}{ Weight (g) } & \multirow{3}{*}{$p$} \\
\hline & & & & \multicolumn{2}{|c|}{$\begin{array}{c}\text { Bread } \\
(n=781)\end{array}$} & \multicolumn{2}{|c|}{$\begin{array}{l}\text { Rice-Based } \\
(n=393)\end{array}$} & \multicolumn{2}{|c|}{$\begin{array}{l}\text { Ramen } \\
(n=99)\end{array}$} & \multicolumn{2}{|c|}{$\begin{array}{l}\text { Udon/Soba } \\
(n=125)\end{array}$} & \multicolumn{2}{|c|}{$\begin{array}{l}\text { Sushi/Rice } \\
\text { Bowl Dishes } \\
\quad(n=106)\end{array}$} & \\
\hline & & $n$ & $\%$ & Mean & SE & Mean & SE & Mean & SE & Mean & SE & Mean & SE & \\
\hline 48 & Japanese and Chinese tea & 968 & 63.9 & $67.3^{a}$ & 2.8 & $320.2^{b}$ & 6.9 & $153.0^{\mathrm{c}}$ & 15.2 & $142.5^{c}$ & 12.3 & $159.3^{c}$ & 14.3 & $<0.0001$ \\
\hline 1 & Rice & 799 & 52.7 & $105.3^{\mathrm{a}}$ & 3.6 & $148.9^{b}$ & 5.0 & $19.0^{\mathrm{c}}$ & 5.8 & $24.9^{c}$ & 5.8 & $0.0^{\mathrm{c}}$ & 0.0 & $<0.0001$ \\
\hline 22 & Raw or boiled vegetable dishes & 437 & 28.8 & $22.5^{\mathrm{a}}$ & 1.7 & $25.9^{\mathrm{a}}$ & 2.6 & $5.7^{\mathrm{b}}$ & 3.3 & $9.7^{\mathrm{b}}$ & 2.5 & $16.3^{\mathrm{ab}}$ & 3.8 & $<0.0001$ \\
\hline 55 & Miso soup & 375 & 24.8 & $53.8^{\mathrm{a}}$ & 3.2 & $51.9^{\mathrm{a}}$ & 4.8 & $0.0^{\mathrm{b}}$ & 0.0 & $4.0^{\mathrm{b}}$ & 2.4 & $66.2^{\mathrm{a}}$ & 8.9 & $<0.0001$ \\
\hline 27 & Fruit/fruit dishes & 326 & 21.5 & 16.2 & 1.2 & 14.1 & 1.7 & 8.0 & 2.5 & 10.9 & 2.5 & 16.7 & 3.8 & 0.12 \\
\hline 21 & Japanese-style vegetable side dishes & 322 & 21.3 & $14.7^{\mathrm{a}}$ & 1.1 & $15.0^{\mathrm{a}}$ & 1.5 & $4.0^{\mathrm{b}}$ & 1.6 & $9.2^{a b}$ & 2.4 & $16.7^{\mathrm{a}}$ & 4.0 & 0.005 \\
\hline 58 & Tsukudani/pickles & 262 & 17.3 & $2.8^{\mathrm{ab}}$ & 0.3 & $4.1^{\mathrm{a}}$ & 0.6 & $2.2^{a b}$ & 0.8 & $0.7^{\mathrm{b}}$ & 0.4 & $3.1^{\mathrm{ab}}$ & 0.8 & 0.006 \\
\hline 47 & Coffee/tea & 251 & 16.6 & $49.2^{\mathrm{a}}$ & 3.6 & $14.1^{\mathrm{b}}$ & 2.8 & $25.5^{\mathrm{ab}}$ & 7.4 & $23.4^{b}$ & 5.6 & $17.5^{\mathrm{b}}$ & 6.2 & $<0.0001$ \\
\hline 40 & Egg dishes & 211 & 13.9 & $8.4^{\mathrm{a}}$ & 0.9 & $14.8^{\mathrm{b}}$ & 1.5 & $0.5^{c}$ & 0.5 & $2.1^{\mathrm{ac}}$ & 1.3 & $4.9^{\mathrm{ac}}$ & 3.6 & $<0.0001$ \\
\hline 16 & Potato dishes & 197 & 13.0 & $12.9^{a b}$ & 1.4 & $16.6^{\mathrm{a}}$ & 2.8 & $0.9^{\mathrm{c}}$ & 0.7 & $3.0^{b c}$ & 1.4 & $5.3^{\mathrm{ac}}$ & 2.3 & 0.0002 \\
\hline 38 & Deep-fried meat dishes & 171 & 11.3 & $11.4^{\mathrm{ab}}$ & 1.3 & $17.1^{\mathrm{a}}$ & 2.5 & $0.8^{\mathrm{bc}}$ & 0.6 & $2.6^{\mathrm{bc}}$ & 1.5 & $0.5^{\mathrm{c}}$ & 0.5 & $<0.0001$ \\
\hline 23 & Simmered vegetable dishes & 171 & 11.3 & $11.4^{\mathrm{ab}}$ & 1.4 & $15.4^{\mathrm{a}}$ & 2.2 & $0.0^{\mathrm{c}}$ & 0.0 & $2.8^{\mathrm{bc}}$ & 1.5 & $4.6^{\mathrm{ac}}$ & 2.1 & 0.0001 \\
\hline 30 & Grilled or stir-fried seafood dishes & 167 & 11.0 & $9.7^{\mathrm{ab}}$ & 1.1 & $12.8^{\mathrm{a}}$ & 1.8 & $0.0^{\mathrm{c}}$ & 0.0 & $1.4^{\mathrm{c}}$ & 0.9 & $3.2^{b c}$ & 1.6 & $<0.0001$ \\
\hline 10 & Udon/soba & 166 & 11.0 & $3.7^{\mathrm{a}}$ & 1.0 & $9.5^{\mathrm{a}}$ & 2.3 & $0.0^{\mathrm{a}}$ & 0.0 & $466.8^{\mathrm{b}}$ & 14.1 & $11.0^{\mathrm{a}}$ & 5.8 & $<0.0001$ \\
\hline 56 & Western or Chinese soup & 144 & 9.5 & $24.4^{\mathrm{a}}$ & 2.7 & $15.0^{\mathrm{ab}}$ & 2.8 & $2.6^{\mathrm{b}}$ & 2.4 & $4.2^{\mathrm{b}}$ & 2.5 & $13.5^{\mathrm{ab}}$ & 5.1 & 0.0003 \\
\hline 5 & Sushi/rice bowl dishes & 140 & 9.2 & $2.4^{\mathrm{a}}$ & 0.7 & $4.8^{\mathrm{a}}$ & 1.6 & $1.1^{\mathrm{a}}$ & 1.1 & $11.2^{\mathrm{a}}$ & 4.2 & $405.2^{b}$ & 15.8 & $<0.0001$ \\
\hline 9 & Sweet bread/savoury bread & 122 & 8.1 & $17.3^{\mathrm{a}}$ & 1.9 & $6.8^{\mathrm{b}}$ & 1.7 & $3.6^{\mathrm{b}}$ & 2.2 & $4.6^{\mathrm{b}}$ & 2.3 & $1.4^{\mathrm{b}}$ & 1.2 & $<0.0001$ \\
\hline 12 & Ramen & 114 & 7.5 & $2.2^{\mathrm{a}}$ & 0.8 & $2.8^{\mathrm{a}}$ & 1.4 & $505.2^{b}$ & 17.6 & $0.0^{\mathrm{a}}$ & 0.0 & $6.1^{\mathrm{a}}$ & 4.3 & $<0.0001$ \\
\hline 24 & Grilled or stir-fried vegetable dishes & 113 & 7.5 & $10.1^{\mathrm{a}}$ & 1.5 & $4.9^{a b}$ & 1.1 & $2.8^{\mathrm{ab}}$ & 1.7 & $2.2^{\mathrm{ab}}$ & 1.6 & $0.6^{\mathrm{b}}$ & 0.6 & 0.002 \\
\hline 6 & Rice ball & 105 & 6.9 & $12.8^{\mathrm{ab}}$ & 2.0 & $14.2^{\mathrm{ab}}$ & 2.9 & $18.2^{a b}$ & 5.5 & $22.8^{\mathrm{a}}$ & 5.8 & $0.0^{\mathrm{b}}$ & 0.0 & 0.03 \\
\hline 44 & Ice cream/jelly/pudding/cake & 101 & 6.7 & 5.3 & 0.9 & 4.3 & 1.0 & 10.7 & 4.0 & 5.7 & 2.1 & 7.1 & 2.1 & 0.19 \\
\hline 32 & Fish jelly product dishes & 97 & 6.4 & 4.7 & 0.9 & 5.8 & 1.6 & 2.8 & 1.6 & 1.7 & 0.9 & 2.2 & 1.8 & 0.42 \\
\hline 18 & Tofu dishes & 94 & 6.2 & 9.1 & 1.4 & 7.5 & 1.8 & 3.1 & 1.6 & 1.0 & 1.0 & 7.8 & 3.1 & 0.13 \\
\hline 39 & Processed meat dishes & 89 & 5.9 & 2.3 & 0.4 & 4.4 & 0.8 & 1.7 & 1.0 & 0.9 & 0.9 & 1.2 & 1.2 & 0.02 \\
\hline 31 & Deep-fried fish dishes & 86 & 5.7 & 5.5 & 0.9 & 4.7 & 1.1 & 1.5 & 1.1 & 2.3 & 1.5 & 0.2 & 0.2 & 0.06 \\
\hline 42 & Yogurt/cheese & 78 & 5.1 & 4.6 & 0.8 & 2.2 & 0.7 & 3.5 & 1.7 & 5.1 & 1.8 & 5.0 & 2.0 & 0.28 \\
\hline 35 & Gyoza dumplings/shumai/meatballs & 72 & 4.8 & 2.0 & 0.4 & 3.9 & 1.0 & 3.7 & 1.7 & 0.0 & 0.0 & 0.6 & 0.6 & 0.02 \\
\hline
\end{tabular}


Table 2. Cont.

\begin{tabular}{|c|c|c|c|c|c|c|c|c|c|c|c|c|c|c|}
\hline \multirow[b]{4}{*}{ ID } & \multirow{4}{*}{$\begin{array}{l}\text { Dish Group } \\
\text { Name }\end{array}$} & \multicolumn{2}{|c|}{$\begin{array}{l}\text { All Lunches } \\
(n=1515)\end{array}$} & \multicolumn{11}{|c|}{$\begin{array}{l}\text { Lunches Included in Cluster Analysis }{ }^{3} \\
\qquad(n=1504)\end{array}$} \\
\hline & & \multirow{2}{*}{\multicolumn{2}{|c|}{ Frequency }} & \multicolumn{10}{|c|}{ Weight (g) } & \multirow{3}{*}{$p$} \\
\hline & & & & \multicolumn{2}{|c|}{$\begin{array}{c}\text { Bread } \\
(n=781)\end{array}$} & \multicolumn{2}{|c|}{$\begin{array}{l}\text { Rice-Based } \\
(n=393)\end{array}$} & \multicolumn{2}{|c|}{$\begin{array}{l}\text { Ramen } \\
(n=99)\end{array}$} & \multicolumn{2}{|c|}{$\begin{array}{l}\text { Udon/Soba } \\
(n=125)\end{array}$} & \multicolumn{2}{|c|}{$\begin{array}{l}\text { Sushi/Rice } \\
\text { Bowl Dishes } \\
\quad(n=106)\end{array}$} & \\
\hline & & $n$ & $\%$ & Mean & SE & Mean & SE & Mean & SE & Mean & SE & Mean & SE & \\
\hline 3 & Curry and rice/omelette rice & 64 & 4.2 & $26.8^{\mathrm{a}}$ & 4.0 & $13.9^{\mathrm{ab}}$ & 3.8 & $2.5^{\mathrm{ab}}$ & 2.5 & $0.0^{\mathrm{b}}$ & 0.0 & $0.0^{\mathrm{b}}$ & 0.0 & 0.0004 \\
\hline 36 & Simmered meat with seasoning & 60 & 4.0 & 9.4 & 1.7 & 6.0 & 1.8 & 0.0 & 0.0 & 0.0 & 0.0 & 0.0 & 0.0 & 0.007 \\
\hline 43 & Japanese confectionery & 57 & 3.8 & 2.2 & 0.5 & 1.5 & 0.7 & 3.3 & 1.7 & 0.7 & 0.6 & 1.7 & 0.8 & 0.56 \\
\hline 29 & Seafood dishes (steamed/boiled/ simmered) & 56 & 3.7 & $4.7^{\mathrm{ab}}$ & 0.9 & $8.9^{\mathrm{a}}$ & 2.2 & $0.0^{\mathrm{ab}}$ & 0.0 & $0.0^{\mathrm{b}}$ & 0.0 & $2.7^{\mathrm{ab}}$ & 2.4 & 0.009 \\
\hline 8 & Plain bread & 53 & 3.5 & $5.5^{\mathrm{a}}$ & 0.9 & $1.0^{\mathrm{b}}$ & 0.4 & $0.7^{\mathrm{ab}}$ & 0.5 & $0.2^{b}$ & 0.2 & $0.0^{\mathrm{b}}$ & 0.0 & $<0.0001$ \\
\hline 11 & Pasta & 50 & 3.3 & 12.7 & 2.5 & 10.7 & 3.0 & 0.0 & 0.0 & 0.0 & 0.0 & 4.4 & 4.4 & 0.07 \\
\hline 4 & Fried rice/pilaf/doria & 48 & 3.2 & 10.8 & 2.1 & 5.3 & 1.8 & 4.2 & 3.3 & 2.8 & 2.8 & 3.5 & 2.9 & 0.14 \\
\hline 57 & Hot pot dishes & 45 & 3.0 & 11.0 & 2.3 & 10.8 & 2.9 & 0.0 & 0.0 & 1.9 & 1.9 & 5.2 & 3.9 & 0.19 \\
\hline
\end{tabular}

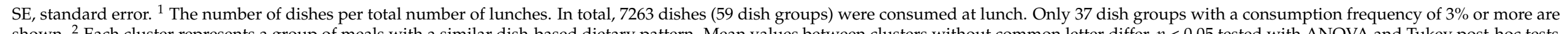

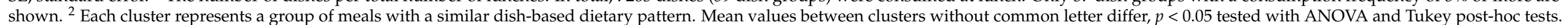

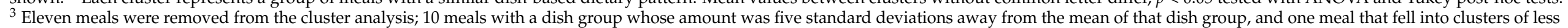
than eight meals when cluster analysis was conducted with a predefined number of 20 clusters.

Table 3. Consumption frequency of dish groups in all dinners ${ }^{1}$ and weight $(\mathrm{g})$ of dish groups in each dietary pattern ${ }^{2}$.

\begin{tabular}{|c|c|c|c|c|c|c|c|c|c|c|}
\hline \multirow[b]{4}{*}{ ID } & \multirow{4}{*}{$\begin{array}{l}\text { Dish Group } \\
\text { Name }\end{array}$} & \multicolumn{2}{|c|}{$\begin{array}{l}\text { All Dinners } \\
(n=1551)\end{array}$} & \multicolumn{7}{|c|}{$\begin{array}{l}\text { Dinners Included in Cluster Analysis }{ }^{3} \\
\qquad(n=1500)\end{array}$} \\
\hline & & \multirow{2}{*}{\multicolumn{2}{|c|}{ Frequency }} & \multicolumn{6}{|c|}{ Weight (g) } & \multirow{3}{*}{$p$} \\
\hline & & & & \multicolumn{2}{|c|}{$\begin{array}{l}\text { Miscellaneous } \\
\quad(n=1211)\end{array}$} & \multicolumn{2}{|c|}{$\begin{array}{l}\text { Meat Dish and Beer } \\
\quad(n=165)\end{array}$} & \multicolumn{2}{|c|}{$\begin{array}{l}\text { Hot Pot Dishes } \\
\quad(n=124)\end{array}$} & \\
\hline & & $n$ & $\%$ & Mean & SE & Mean & SE & Mean & SE & \\
\hline 48 & Japanese and Chinese tea & 909 & 58.6 & $166.3^{\mathrm{a}}$ & 4.8 & $72.1^{\mathrm{b}}$ & 10.2 & $162.7^{\mathrm{a}}$ & 16.1 & $<0.0001$ \\
\hline 1 & Rice & 874 & 56.4 & $106.2^{\mathrm{a}}$ & 3.1 & $62.3^{\mathrm{b}}$ & 7.1 & $102.0^{\mathrm{a}}$ & 10.2 & $<0.0001$ \\
\hline 55 & Miso soup & 464 & 29.9 & $79.7^{\mathrm{a}}$ & 3.7 & $73.4^{\mathrm{a}}$ & 10.0 & $4.0^{\mathrm{b}}$ & 2.3 & $<0.0001$ \\
\hline 22 & Raw or boiled vegetable dishes & 459 & 29.6 & $36.1^{\mathrm{a}}$ & 2.0 & $46.7^{\mathrm{a}}$ & 6.2 & $17.3^{\mathrm{b}}$ & 4.8 & 0.002 \\
\hline 21 & Japanese-style vegetable side dishes & 282 & 18.2 & $15.5^{\mathrm{a}}$ & 1.1 & $18.2^{\mathrm{a}}$ & 3.8 & $6.8^{\mathrm{b}}$ & 2.1 & 0.03 \\
\hline
\end{tabular}


Table 3. Cont

\begin{tabular}{|c|c|c|c|c|c|c|c|c|c|c|}
\hline \multirow[b]{4}{*}{ ID } & \multirow{4}{*}{$\begin{array}{l}\text { Dish Group } \\
\text { Name }\end{array}$} & \multirow{3}{*}{\multicolumn{2}{|c|}{$\begin{array}{c}\text { All Dinners } \\
(n=1551)\end{array}$}} & \multicolumn{7}{|c|}{$\begin{array}{l}\text { Dinners Included in Cluster Analysis }{ }^{3} \\
\qquad(n=1500)\end{array}$} \\
\hline & & & & \multicolumn{6}{|c|}{ Weight (g) } & \multirow{3}{*}{$p$} \\
\hline & & & & \multicolumn{2}{|c|}{$\begin{array}{l}\text { Miscellaneous } \\
\quad(n=1211)\end{array}$} & \multicolumn{2}{|c|}{$\begin{array}{l}\text { Meat Dish and Beer } \\
\qquad(n=165)\end{array}$} & \multicolumn{2}{|c|}{$\begin{array}{l}\text { Hot Pot Dishes } \\
\quad(n=124)\end{array}$} & \\
\hline & & $n$ & $\%$ & Mean & SE & Mean & SE & Mean & SE & \\
\hline 30 & Grilled or stir-fried seafood dishes & 265 & 17.1 & $17.9^{\mathrm{a}}$ & 1.4 & $18.6^{\mathrm{a}}$ & 3.3 & $5.6^{\mathrm{b}}$ & 2.1 & 0.01 \\
\hline 37 & Grilled or stir-fried meat dishes & 258 & 16.6 & $35.6^{\mathrm{a}}$ & 2.8 & $42.3^{\mathrm{a}}$ & 7.3 & $3.3^{b}$ & 2.2 & 0.0006 \\
\hline 58 & Tsukudani/pickles & 220 & 14.2 & 4.1 & 0.4 & 5.1 & 1.2 & 5.1 & 1.6 & 0.53 \\
\hline 53 & Beer & 206 & 13.3 & $2.3^{\mathrm{a}}$ & 0.7 & $604.8^{b}$ & 24.8 & $35.6^{\mathrm{c}}$ & 11.0 & $<0.0001$ \\
\hline 16 & Potato dishes & 179 & 11.5 & $17.3^{\mathrm{a}}$ & 1.7 & $18.7^{\mathrm{ab}}$ & 5.0 & $4.5^{\mathrm{b}}$ & 2.8 & 0.053 \\
\hline 57 & Hot pot dishes & 173 & 11.2 & $5.9^{\mathrm{a}}$ & 1.0 & $18.2^{\mathrm{a}}$ & 7.2 & $640.5^{b}$ & 21.6 & $<0.0001$ \\
\hline 27 & Fruit/fruit dishes & 169 & 10.9 & 10.3 & 1.0 & 14.7 & 3.6 & 8.0 & 2.9 & 0.24 \\
\hline 38 & Deep-fried meat dishes & 152 & 9.8 & $14.9^{\mathrm{a}}$ & 1.5 & $17.9^{\mathrm{a}}$ & 4.8 & $1.6^{\mathrm{b}}$ & 1.1 & 0.02 \\
\hline 5 & Sushi/rice bowl dishes & 147 & 9.5 & $40.6^{\mathrm{a}}$ & 3.7 & $31.8^{\mathrm{ab}}$ & 8.5 & $4.1^{\mathrm{b}}$ & 2.4 & 0.006 \\
\hline 18 & Tofu dishes & 147 & 9.5 & $20.5^{\mathrm{a}}$ & 2.0 & $7.9^{\mathrm{ab}}$ & 2.8 & $3.4^{\mathrm{b}}$ & 2.3 & 0.003 \\
\hline 24 & Grilled or stir-fried vegetable dishes & 139 & 9.0 & $13.3^{\mathrm{a}}$ & 1.4 & $13.0^{\mathrm{ab}}$ & 3.3 & $1.3^{\mathrm{b}}$ & 1.3 & 0.03 \\
\hline 52 & Japanese sake/shochu & 114 & 7.4 & $17.7^{\mathrm{a}}$ & 3.1 & $73.5^{b}$ & 11.9 & $72.8^{\mathrm{b}}$ & 19.9 & $<0.0001$ \\
\hline 56 & Western or Chinese soup & 113 & 7.3 & $19.0^{\mathrm{a}}$ & 2.1 & $8.5^{\mathrm{ab}}$ & 3.8 & $1.4^{\mathrm{b}}$ & 1.4 & 0.005 \\
\hline 28 & Raw fish dishes & 109 & 7.0 & 6.5 & 0.9 & 9.9 & 2.6 & 7.4 & 3.1 & 0.43 \\
\hline 10 & Udon/soba & 98 & 6.3 & $29.3^{a}$ & 3.4 & $28.5^{\mathrm{ab}}$ & 8.7 & $0.0^{\mathrm{b}}$ & 0.0 & 0.02 \\
\hline 40 & Egg dishes & 97 & 6.3 & 7.5 & 1.0 & 10.2 & 3.1 & 2.0 & 1.5 & 0.13 \\
\hline 17 & Natto (fermented soybeans) & 96 & 6.2 & 3.4 & 0.4 & 1.4 & 0.9 & 2.8 & 0.9 & 0.19 \\
\hline 47 & Coffee/tea & 92 & 5.9 & 15.6 & 1.9 & 4.6 & 2.3 & 9.9 & 3.7 & 0.08 \\
\hline 26 & Mushrooms/seaweed/konnyaku dishes & 86 & 5.5 & 4.7 & 0.7 & 5.8 & 2.2 & 0.8 & 0.5 & 0.15 \\
\hline 35 & Gyoza dumplings/shumai/meatballs & 83 & 5.4 & $7.2^{\mathrm{a}}$ & 1.1 & $15.3^{b}$ & 4.4 & $3.0^{\mathrm{a}}$ & 2.0 & 0.02 \\
\hline 29 & Seafood dishes (steamed/boiled/ simmered) & 82 & 5.3 & 10.1 & 1.4 & 11.8 & 3.9 & 3.4 & 2.2 & 0.29 \\
\hline 36 & Simmered meat with seasoning & 79 & 5.1 & $14.1^{\mathrm{a}}$ & 2.1 & $33.4^{b}$ & 8.8 & $0.0^{\mathrm{a}}$ & 0.0 & 0.0006 \\
\hline 3 & Curry and rice/omelette rice & 78 & 5.0 & $27.6^{\mathrm{a}}$ & 3.6 & $37.4^{\mathrm{a}}$ & 10.7 & $0.0^{\mathrm{b}}$ & 0.0 & 0.03 \\
\hline 54 & Fruit liquor/wine & 76 & 4.9 & $14.6^{\mathrm{a}}$ & 2.4 & $37.4^{\mathrm{b}}$ & 11.6 & $29.8^{a b}$ & 11.9 & 0.009 \\
\hline 31 & Deep-fried fish dishes & 70 & 4.5 & 4.2 & 0.7 & 5.7 & 2.3 & 2.0 & 1.0 & 0.41 \\
\hline 44 & Ice cream/jelly/pudding/cake & 64 & 4.1 & 4.0 & 0.6 & 3.5 & 1.4 & 2.4 & 1.3 & 0.73 \\
\hline 12 & Ramen & 57 & 3.7 & 22.5 & 3.4 & 6.6 & 4.0 & 3.8 & 3.8 & 0.052 \\
\hline
\end{tabular}


Table 3. Cont.

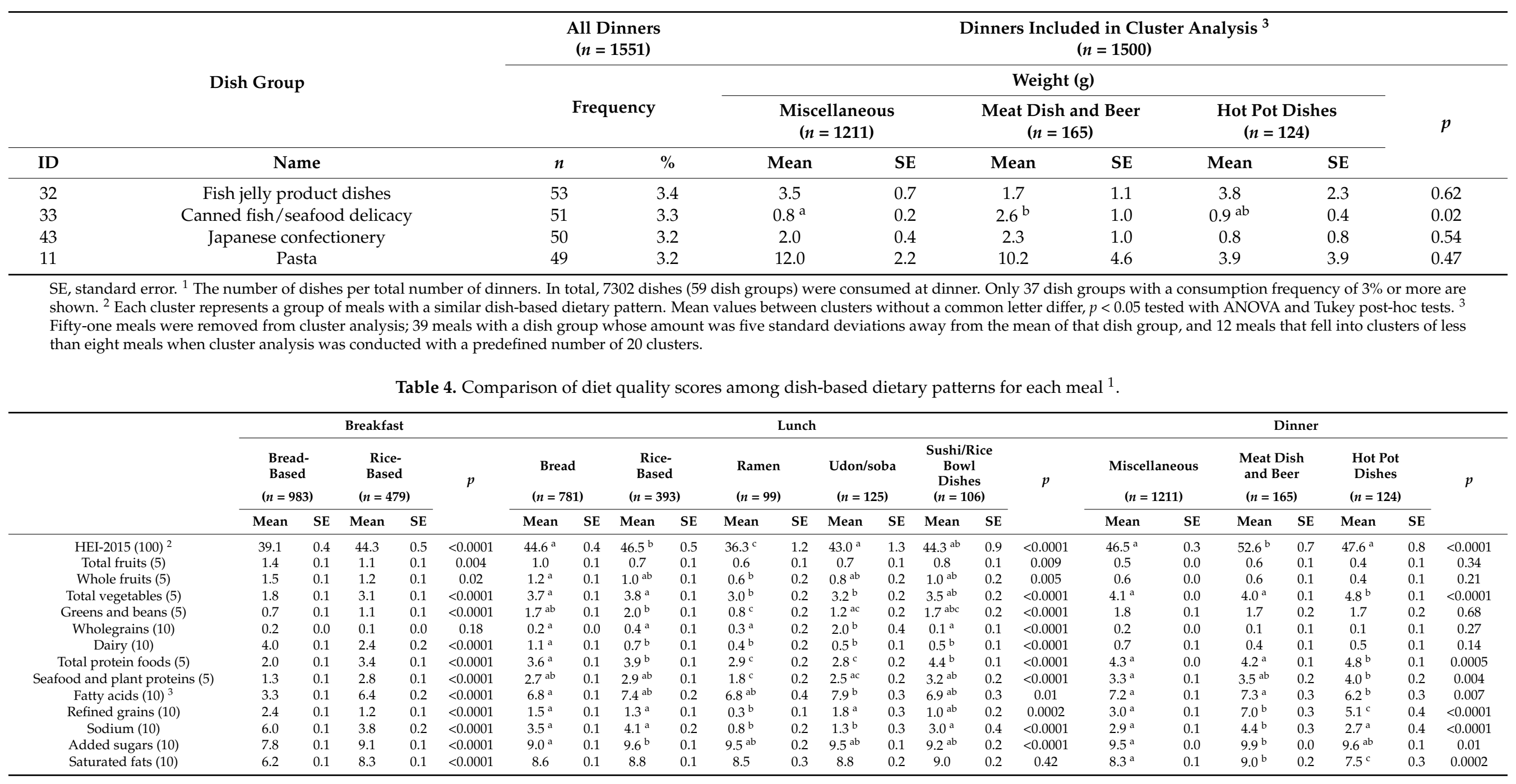


Table 4. Cont.

\begin{tabular}{|c|c|c|c|c|c|c|c|c|c|c|c|c|c|c|c|c|c|c|c|c|c|c|c|}
\hline & \multicolumn{5}{|c|}{ Breakfast } & \multicolumn{11}{|c|}{ Lunch } & \multicolumn{7}{|c|}{ Dinner } \\
\hline & \multicolumn{2}{|c|}{$\begin{array}{c}\text { Bread- } \\
\text { Based } \\
(n=983)\end{array}$} & \multicolumn{2}{|c|}{$\begin{array}{c}\text { Rice- } \\
\text { Based } \\
(n=479)\end{array}$} & $p$ & \multicolumn{2}{|c|}{$\begin{array}{c}\text { Bread } \\
(n=781)\end{array}$} & \multicolumn{2}{|c|}{$\begin{array}{c}\text { Rice- } \\
\text { Based } \\
(n=393)\end{array}$} & \multicolumn{2}{|c|}{$\begin{array}{l}\text { Ramen } \\
(n=99)\end{array}$} & \multicolumn{2}{|c|}{$\begin{array}{l}\text { Udon/soba } \\
\qquad(n=125)\end{array}$} & \multicolumn{2}{|c|}{$\begin{array}{c}\text { Sushi/Rice } \\
\text { Bowl } \\
\text { Dishes } \\
(n=106)\end{array}$} & $p$ & \multicolumn{2}{|c|}{$\begin{array}{l}\text { Miscellaneous } \\
\qquad(n=1211)\end{array}$} & \multicolumn{2}{|c|}{$\begin{array}{c}\text { Meat Dish } \\
\text { and Beer } \\
(n=165)\end{array}$} & \multicolumn{2}{|c|}{$\begin{array}{c}\text { Hot Pot } \\
\text { Dishes } \\
(n=124)\end{array}$} & $p$ \\
\hline NRF9.3 (900) 4 & 421 & 10 & 538 & 8 & $<0.0001$ & $493^{a}$ & 11 & $563^{b}$ & 9 & $348^{c}$ & 24 & $416^{c}$ & 20 & 513 ab & 17 & $<0.0001$ & $536^{\mathrm{a}}$ & 6 & 559 ab & 11 & $582^{b}$ & 14 & 0.02 \\
\hline Protein (100) & 95.2 & 0.4 & 97.5 & 0.4 & 0.0008 & $96.3^{\mathrm{a}}$ & 0.4 & $98.1^{\mathrm{b}}$ & 0.4 & $98.4^{\mathrm{ab}}$ & 0.5 & $96.7 \mathrm{ab}$ & 0.6 & $98.3^{\text {ab }}$ & 0.6 & 0.02 & $98.4^{\mathrm{a}}$ & 0.2 & $95.0^{\mathrm{b}}$ & 1.1 & $99.3^{\mathrm{a}}$ & 0.4 & $<0.0001$ \\
\hline Dietary fibre (100) & 67.1 & 1.0 & 76.5 & 1.2 & $<0.0001$ & $74.1^{\mathrm{a}}$ & 1.0 & $71.5^{\mathrm{a}}$ & 1.4 & $86.6^{\mathrm{b}}$ & 1.8 & $83.3^{\mathrm{b}}$ & 1.7 & $62.8^{\mathrm{c}}$ & 2.8 & $<0.0001$ & $76.7^{\mathrm{a}}$ & 0.8 & $62.9^{\mathrm{b}}$ & 2.2 & $89.0^{\mathrm{c}}$ & 1.7 & $<0.0001$ \\
\hline Vitamin A (100) & 48.7 & 1.1 & 54.2 & 1.6 & 0.005 & $60.5^{a}$ & 1.2 & $60.7^{a}$ & 1.6 & $34.5^{\mathrm{b}}$ & 3.3 & $45.1^{\mathrm{b}}$ & 3.2 & $59.1^{\mathrm{a}}$ & 3.2 & $<0.0001$ & $59.6^{\mathrm{a}}$ & 1.0 & $48.1^{\mathrm{b}}$ & 2.5 & $63.2^{\mathrm{a}}$ & 3.2 & $<0.0001$ \\
\hline Vitamin C (100) & 52.1 & 1.4 & 75.6 & 1.6 & $<0.0001$ & $76.4^{\mathrm{a}}$ & 1.2 & $86.0^{\mathrm{b}}$ & 1.4 & $62.3^{c}$ & 3.8 & $62.0^{\mathrm{c}}$ & 3.4 & $72.5^{\mathrm{ac}}$ & 3.1 & $<0.0001$ & $81.1^{\mathrm{a}}$ & 0.9 & $75.8^{\mathrm{a}}$ & 2.3 & $91.6^{\mathrm{b}}$ & 1.8 & $<0.0001$ \\
\hline Calcium (100) & 76.9 & 0.9 & 74.8 & 1.3 & 0.19 & $60.7^{a b}$ & 1.0 & $58.9^{\mathrm{ab}}$ & 1.4 & $65.5^{\mathrm{ab}}$ & 2.5 & $65.9^{\text {a }}$ & 2.6 & $55.2^{\mathrm{b}}$ & 2.5 & 0.009 & $64.0^{\mathrm{a}}$ & 0.8 & $57.4^{\mathrm{b}}$ & 2.0 & $81.0^{\mathrm{c}}$ & 2.0 & $<0.0001$ \\
\hline Iron (100) & 82.9 & 0.8 & 94.4 & 0.7 & $<0.0001$ & $92.2^{\mathrm{a}}$ & 0.6 & $96.1^{\mathrm{b}}$ & 0.6 & $92.9 \mathrm{ab}$ & 1.2 & 94.6 ab & 0.9 & $94.5^{\mathrm{ab}}$ & 1.1 & 0.001 & $95.4^{\mathrm{a}}$ & 0.4 & $89.2^{\mathrm{b}}$ & 1.5 & $98.6^{\mathrm{c}}$ & 0.5 & $<0.0001$ \\
\hline Potassium (100) & 85.7 & 0.7 & 85.9 & 1.0 & 0.84 & $84.7^{\text {a }}$ & 0.8 & $85.0^{\mathrm{a}}$ & 1.0 & $79.1 \mathrm{ab}$ & 2.4 & $71.5^{\mathrm{b}}$ & 2.3 & $84.1^{\mathrm{a}}$ & 1.8 & $<0.0001$ & $89.6^{\mathrm{a}}$ & 0.5 & $86.5^{\mathrm{a}}$ & 1.4 & $97.2^{\mathrm{b}}$ & 0.9 & $<0.0001$ \\
\hline Magnesium (100) & 92.5 & 0.5 & 92.7 & 0.7 & 0.81 & 89.5 & 0.7 & 89.2 & 0.9 & 86.5 & 1.6 & 90.8 & 1.3 & 91.4 & 1.4 & 0.27 & $93.4^{\mathrm{a}}$ & 0.4 & $94.4^{\mathrm{ab}}$ & 0.9 & $97.9^{\mathrm{b}}$ & 0.7 & 0.002 \\
\hline Added sugars ( - ) & 141.8 & 10.2 & 46.1 & 5.1 & $<0.0001$ & $66.5^{\text {a }}$ & 7.7 & $22.8^{\mathrm{b}}$ & 3.6 & $27.3 \mathrm{ab}$ & 6.8 & $26.6 \mathrm{ab}$ & 6.6 & $45.6^{\mathrm{ab}}$ & 10.5 & 0.0001 & $28.9^{\mathrm{a}}$ & 3.1 & $8.0^{\mathrm{b}}$ & 2.0 & $20.4 \mathrm{ab}$ & 5.1 & 0.03 \\
\hline Saturated fats (-) & 63.9 & 2.5 & 28.1 & 2.5 & $<0.0001$ & 23.6 & 1.6 & 20.7 & 2.2 & 22.7 & 4.0 & 17.9 & 3.2 & 16.9 & 2.9 & 0.39 & $27.2^{\mathrm{a}}$ & 1.3 & $17.8^{\mathrm{b}}$ & 3.2 & $41.8^{\mathrm{c}}$ & 5.3 & $<0.0001$ \\
\hline Sodium $(-)$ & 55.1 & 3.0 & 97.3 & 4.9 & $<0.0001$ & $97.3^{\mathrm{a}}$ & 4.6 & $85.4^{\mathrm{a}}$ & 5.0 & $228.9^{b}$ & 15.9 & $168.1^{c}$ & 9.5 & $91.9^{\mathrm{a}}$ & 7.8 & $<0.0001$ & $113.5^{\mathrm{a}}$ & 3.4 & $71.8^{\mathrm{b}}$ & 5.8 & $117.9^{\mathrm{a}}$ & 9.7 & $<0.0001$ \\
\hline
\end{tabular}

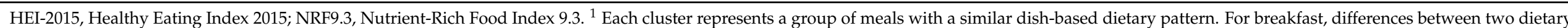

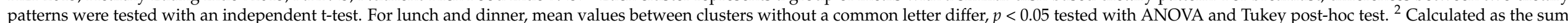

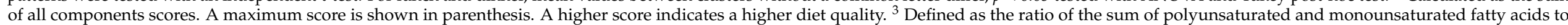

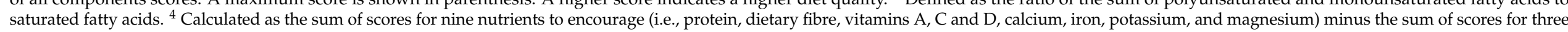

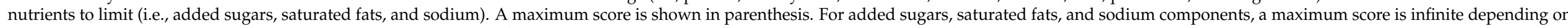
the amount. A higher score indicates a higher diet quality, except for added sugars, saturated fats, and sodium components, for which a higher score indicates a lower diet quality. 


\section{Discussion}

In the present study, we identified different numbers of dietary patterns for each eating occasion: two for breakfast, five for lunch, and three for dinner. Each of the dietary patterns differed in amount and type of dish groups as well as diet quality. Although there have been several studies that investigated meal-specific dietary patterns at breakfast level or main-meal level [4,9-13], they have analysed meals at the level of single foods or food groups. Moreover, overall diet quality has not been assessed for each dietary pattern. To our knowledge, this is the first study to investigate dish-based dietary patterns and the diet quality of each pattern for breakfast, lunch, and dinner.

Previous studies have shown that some food groups were commonly consumed at all three main meals in Japan, such as rice, vegetables, tea, and coffee, whereas the consumption frequency and amount of many other food groups differed among eating occasions [5,8,11]. Similarly, in this study, there are some common dish groups in the top five most frequently consumed items on each eating occasion (i.e., Japanese and Chinese tea, rice, and miso soup), while the consumption frequency of many dish groups differed among eating occasions. For instance, milk/soymilk, cocoa/milk beverage, and vegetable and fruit juice were frequently consumed at breakfasts, fried rice/pilaf/doria was frequently consumed at lunch, and raw fish dishes and alcoholic beverages (beer, Japanese sake/shochu, and fruit liquor/wine) were frequently consumed at dinner. Consequently, separate dietary patterns with different levels of diet quality scores were derived for each eating occasion. A previous study conducted in Germany has also shown that the consumption frequency of food groups varied across eating occasions [4]. These results support the appropriateness and importance of dietary analysis focusing on each eating occasion.

The number of dietary patterns identified in this study was the smallest in breakfast. This is consistent with a previous study conducted in Brazil [13], whereas other studies have derived equal numbers of dietary patterns for each eating occasion (two patterns in China and four patterns in Japan) [11,12]. Our results showed that dietary patterns at breakfast and lunch were mainly characterised by staple foods (i.e., bread, rice, or noodles). In addition, several dietary patterns were similar between breakfast and lunch, namely the 'bread-based' pattern for breakfast and the 'bread' pattern for lunch, and the 'rice-based' patterns for breakfast and lunch. The other dietary patterns for lunch were characterised by ramen, udon/soba, and sushi/rice bowl dishes, which were rarely eaten at breakfast (consumption frequency $<1.2 \%$ ). A previous study on food group intake has also identified dietary patterns characterised by rice or bread for breakfast, and those characterised by bread or noodles for lunch among Japanese adults [11]. These results reveal distinctive dietary patterns in relation to eating occasions among Japanese people. Meanwhile, for dinner, although the 'meat dish and beer' pattern was characterised by a relatively small content of rice, none of the dietary patterns were characterised by a large amount of staple foods. Rather, dietary patterns at dinner were particularly characterised by other dish groups, such as alcoholic beverages and hot pot dishes.

For breakfast, the number of meals of the 'bread-based' pattern was twice that of the 'rice-based' pattern. Previous studies conducted in Mexico and the United States have also shown several dietary patterns for breakfast characterised by bread, while there has been no dietary pattern characterised by rice $[9,10]$. The 'rice-based' pattern in breakfast had similar characteristics to the 'Japanese' pattern identified in a previous study, which was characterised by larger intakes of mushrooms, seaweeds, potatoes, vegetables, pickles, pulses, seasonings, fruits, and fish and shellfish [44]. Both the HEI2015 and NRF9.3 showed that overall diet quality was higher in the 'rice-based' pattern than the 'bread-based' pattern at breakfast. The 'rice-based' pattern had higher HEI-2015 component scores for total vegetable, greens and beans, total protein foods, and seafood and plant proteins. This may be due to large amounts of Japanese-style vegetable side dishes: mushrooms/seaweed/konnyaku dishes, natto (fermented soybeans), egg dishes, grilled or stir-fried seafood dishes, and canned fish/seafood delicacy. However, both diet quality measures showed that the 'rice-based' pattern in breakfast contained higher 
sodium than the 'bread-based' pattern. A previous study analysing sodium sources in the same population has revealed that the top contributor to total sodium intake was seasonings, followed by fish and shellfish [16]. These foods are the main ingredients of the dish groups characterising the 'rice-based' pattern, such as miso soup, tsukudani (fish, meat, or seaweeds simmered in soy sauce) and pickles, and canned fish/seafood delicacy (including salted fish and shellfish). Traditionally, rice is eaten together with such salty foods in Japan. Therefore, reducing these salty dishes would be a key to improving diet quality in the 'rice-based' pattern. On the other hand, while the 'bread-based' pattern had less sodium content, this pattern contained higher amounts of added sugars and saturated fats than the 'rice' pattern. This may be because this pattern was characterised by dish groups that are the major sources of sugars and fats, i.e., coffee/tea (including those with sugar and milk), plain bread (including those with butter, margarine, or jam), sweet bread/savoury bread, milk/soymilk, and ice cream/jelly/pudding/cake.

As with the breakfast dietary pattern, the predominant pattern in lunch was the 'bread' pattern, followed by the 'rice-based' pattern. In comparison, the numbers of meals of the 'udon/soba', 'sushi/ricebowl dishes', and 'ramen' patterns were small. The 'rice-based' pattern had a relatively high overall diet quality assessed by the HEI-2015 and NRF9.3. The 'rice-based' pattern in lunch had similar sodium content to that in breakfast. However, sodium content was much higher in the 'ramen' and 'udon/soba' patterns. This may be attributable to the noodle itself and noodle soup [16]. The 'ramen' pattern had the lowest overall diet quality assessed by HEI-2015 among lunch dietary patterns. Compared to the 'rice-based' pattern, the 'ramen' pattern had lower amounts of dish groups including egg dishes, potato dishes, grilled or stir-fried meat dishes, simmered vegetable dishes, and grilled or stir-fried seafood dishes. As such, it seems that ramen is not eaten in combination with main or side dishes consisting of a variety of foods, possibly resulting in the low HEI-2015 component scores for total vegetables, greens and beans, total protein foods, and seafood and plant proteins. In addition, the 'ramen' pattern also had lower overall dietary quality assessed by NRF9.3 among the lunch patterns, and the same was true with the 'udon/soba' pattern, another noodle-based dietary pattern. Nevertheless, the 'udon/soba' pattern had the highest HEI component scores of wholegrain, mainly because of soba (buckwheat noodle).

For dinner, most meals ( $81 \%$ ) were categorised into the 'miscellaneous' dietary pattern. This pattern contained a larger amount of rice and had the lowest HEI component score for refined grains. On the other hand, the 'meat dish and beer' pattern, which had the smallest amount of rice, had the highest component score for refined grain, and also had the highest HEI total score. The 'meat dish and beer' pattern was also characterised by the largest amounts of meat dishes, including gyoza dumplings/shumai/meatballs and simmered meat with seasoning. Nevertheless, it showed the highest quality (namely, lowest content) for saturated fats in both HEI-2015 and NRF9.3. Rather, the 'hot pot dishes' pattern had a lower score for saturated fats in both diet quality measures. This may be because hot pot dishes (including shabu-shabu and sukiyaki) consist of a lot of meat (thinly sliced pork or beef). Moreover, since hot pot dishes include various kinds of vegetables, the 'hot pot dishes' pattern had the highest HEI-2015 component scores for total vegetables and total protein foods, despite the smallest amounts of stir-fried meat or seafood dishes as well as vegetable dishes. The 'hot pot dishes' pattern also had relatively high diet quality, as assessed by NRF 9.3 .

A strength of this study is its examination of meal-specific dietary patterns at the level of mixed dishes, using detailed dietary information obtained from a $4 \mathrm{~d}$ DR. Importantly, this approach enabled us to reveal dish-based dietary patterns that could not be identified when looking at individual food intake. Since mixed dishes are the state of foods closer to that in a real eating situation, dish-based dietary pattern analysis focusing on eating occasions would provide an important basis for elucidating people's dietary behaviours and developing practical dietary advice. For instance, it would be important to reduce salty dishes for rice-based meals, and sugary or fatty dishes for bread-based meals, for 
better diet quality at breakfast. In addition, when eating ramen for lunch, increasing the variety of foods by adding side dishes would be important. Such practical advice can be useful to develop public health policy to facilitate healthier diet choices. Moreover, we used cluster analysis to group meals based on the similarity in the amounts of dish groups, which enabled us to clarify dish groups consumed together at meals. However, given that most dinners were classified into the 'miscellaneous' dietary pattern, dietary patterns may not have been clearly separated for dinner, possibly due to the small sample size. Thus, increasing sample size or using other approaches, such as principal component analysis, should be considered in future research.

Several limitations of this study should be mentioned. First, the DR was obtained over four days per person, which may have reduced the variation in types of dish groups and dietary patterns. Second, since the DR was obtained only in winter, seasonal variation in mixed dishes was not reflected in dietary data. For example, hot pot dishes, which characterised one of the dietary patterns for dinner, are not frequently eaten in other seasons in general. Given the seasonal variation in dietary intake [45], dish-based dietary patterns should be investigated using dietary data over four seasons in future analysis. Third, selfreported dietary information is subject to both random and systematic errors [46,47], which may have had an influence on deriving dietary patterns [3]. Although we used energyadjusted values for diet quality measures, the results should be interpreted with caution. Fourth, since the NRF9.3 was calculated based on the RDVs for men aged 30-49 years uniformly (except for added sugar), the nutritional quality of individual meals may have not been accurately evaluated. However, this may not have greatly influenced the study results, given that NRF9.3 reflects the nutrient density of the diet. Moreover, NRF9.3 provided generally similar results to HEI-2015, which was not calculated using the RDVs. Fifth, cluster analysis is hindered by subjective analytical decisions [3], including the number (and grouping) of dish groups, the definition of eating occasions, the form of the input variables, the number of cluster solutions, as well as clustering methods. In addition, although we assessed the reproducibility of the clusters in a random half of the samples, the reproducibility of cluster solutions does not guarantee validity [48]. Sixth, the study participants were not randomly selected, but were healthy volunteers, likely more healthconscious than the general population. Finally, although beyond the scope of the study, we did not examine the differences in dietary patterns related to individual characteristics such as sex, age, and obesity status. Moreover, we did not assess the association between the dish-based dietary patterns of individual meals and the overall dietary quality at the individual level, which was beyond the scope of this study. Thus, it would be of interest to investigate the association of dish-based dietary patterns of individual meals with participant characteristics, or overall diet quality, in future research.

\section{Conclusions}

In conclusion, using a $4 \mathrm{~d}$ DR obtained from Japanese adults, we investigated dishbased dietary patterns for breakfast, lunch, and dinner, and assessed their diet quality. The results showed distinctive dietary patterns: two patterns for breakfast, five for lunch, and three for dinner, which may serve to help us understand the meal-specific dietary choices of Japanese people. Moreover, this study provided insight into the health aspects of dietary patterns at each eating occasion. The diet scoring of meal- and dish-based dietary patterns would help to provide specific dietary advice in the meal context so that people can modify their dietary behaviour. Future studies with increased sample sizes and different statistical approaches are required to obtain further insight into dish-based dietary patterns.

Supplementary Materials: The following are available online at https:/ /www.mdpi.com/2072-664 3/13/1/67/s1, Table S1: Dish groups used in this study, Table S2: Reference daily values used to calculate NRF9.3, Table S3: Food groups used in this study, Table S4: Comparison of weight (g) of food groups among dish-based dietary patterns for each meal. 
Author Contributions: Conceptualisation, N.S. and K.M.; methodology, N.S. and K.M.; formal analysis, N.S. and K.M.; investigation, N.S. and K.M.; data curation, K.A., S.M. and S.S.; writingoriginal draft preparation, N.S. and K.M.; writing-review and editing, N.S. and K.M.; visualisation, N.S. and K.M.; supervision, K.M.; project administration, N.S. and K.M.; funding acquisition, S.S. All authors have read and agreed to the published version of the manuscript.

Funding: This research was funded by the Ministry of Health, Labour and Welfare, Japan, a Health and Labour Sciences Research Grant (number H23-Jyunkankitou (seishuu)-ippan-001).

Institutional Review Board Statement: The study was conducted according to the guidelines of the Declaration of Helsinki, and approved by the Ethics Committee of the University of Tokyo, Faculty of Medicine (protocol code 10005 and date of approval: 9 January 2013.

Informed Consent Statement: Informed consent was obtained from all subjects involved in the study.

Data Availability Statement: The data presented in this study are available on request from the corresponding author. The data are not publicly available due to privacy or ethical restrictions.

Acknowledgments: The authors are grateful to the participants and local staff for their participation in this study.

Conflicts of Interest: The authors declare no conflict of interest. The funders had no role in the design of the study; in the collection, analyses, or interpretation of data; in the writing of the manuscript, or in the decision to publish the results.

\section{References}

1. Hu, F.B. Dietary pattern analysis: A new direction in nutritional epidemiology. Curr. Opin. Lipidol. 2002, 13, 3-9. [CrossRef] [PubMed]

2. Jacobs, D.R.; Steffen, L.M. Nutrients, foods, and dietary patterns as exposures in research: A framework for food synergy. Am. J. Clin. Nutr. 2003, 78, 508-513. [CrossRef] [PubMed]

3. Kant, A.K. Dietary patterns and health outcomes. J. Am. Diet. Assoc. 2004, 104, 615-635. [CrossRef] [PubMed]

4. Schwedhelm, C.; Iqbal, K.; Knüppel, S.; Schwingshackl, L.; Boeing, H. Contribution to the understanding of how principal component analysis-derived dietary patterns emerge from habitual data on food consumption. Am. J. Clin. Nutr. 2018, 107, 227-235. [CrossRef]

5. Murakami, K.; Livingstone, M.B.E.; Sasaki, S. Establishment of a meal coding system for the characterization of meal-based dietary patterns in Japan. J. Nutr. 2017, 147, 2093-2101. [CrossRef]

6. Woolhead, C.; Gibney, M.J.; Walsh, M.C.; Brennan, L.; Gibney, E.R. A generic coding approach for the examination of meal patterns. Am. J. Clin. Nutr. 2015, 102, 316-323. [CrossRef]

7. Hearty, Á.P.; Gibney, M.J. Analysis of meal patterns with the use of supervised data mining techniques-Artificial neural networks and decision trees. Am. J. Clin. Nutr. 2008, 88, 1632-1642. [CrossRef]

8. Murakami, K.; Livingstone, M.B.E.; Shinozaki, N.; Sugimoto, M.; Fujiwara, A.; Masayasu, S.; Sasaki, S. Food combinations in relation to the quality of overall diet and individual meals in Japanese adults: A nationwide study. Nutrients 2020, 12, 327. [CrossRef]

9. Afeiche, M.C.; Taillie, L.S.; Hopkins, S.; Eldridge, A.L.; Popkin, B.M. Breakfast dietary patterns among Mexican children are related to total-day diet quality. J. Nutr. 2017, 147, 404-412. [CrossRef]

10. O'Neil, C.E.; Nicklas, T.A.; Fulgoni, V.L. Nutrient intake, diet quality, and weight/adiposity parameters in breakfast patterns compared with no breakfast in adults: National health and nutrition examination survey 2001-2008. J. Acad. Nutr. Diet. 2014, 114, S27-S43. [CrossRef]

11. Murakami, K.; Livingstone, M.B.E.; Sasaki, S. Meal-specific dietary patterns and their contribution to overall dietary patterns in the Japanese context: Findings from the 2012 National Health and Nutrition Survey, Japan. Nutrition 2019, 59, 108-115. [CrossRef] [PubMed]

12. Shi, Z.; Riley, M.; Taylor, A.; Noakes, M. Meal-specific food patterns and the incidence of hyperglycemia in a Chinese adult population. Br. J. Nutr. 2017, 118, 53-59. [CrossRef] [PubMed]

13. De Oliveira Santos, R.; Fisberg, R.M.; Marchioni, D.M.; Troncoso Baltar, V. Dietary patterns for meals of Brazilian adults. Br. J. Nutr. 2015, 114, 822-828. [CrossRef] [PubMed]

14. Date, C.; Yamaguchi, M.; Tanaka, H. Development of a food frequency questionnaire in Japan. J. Epidemiol. 1996, 6, 131-136. [CrossRef]

15. Asakura, K.; Uechi, K.; Sasaki, Y.; Masayasu, S.; Sasaki, S. Estimation of sodium and potassium intakes assessed by two $24 \mathrm{~h}$ urine collections in healthy Japanese adults: a nationwide study. Br. J. Nutr. 2014, 112, 1195-1205. [CrossRef]

16. Asakura, K.; Uechi, K.; Masayasu, S.; Sasaki, S. Sodium sources in the Japanese diet: Difference between generations and sexes. Public Health Nutr. 2016, 19, 2011-2023. [CrossRef]

17. Council for Science and Technology; Ministry of Education, Culture, Sports Science and Technology, Japan. Standard Tables of Food Composition in Japan 2015, 7th ed.; Official Gazette Co-operation of Japan: Tokyo, Japan, 2015. 
18. Shinozaki, N.; Murakami, K.; Masayasu, S.; Sasaki, S. Development and simulated validation of a dish composition database for estimating food group and nutrient intakes in Japan. Public Health Nutr. 2019, 22, 2367-2380. [CrossRef]

19. Shinozaki, N.; Murakami, K.; Masayasu, S.; Sasaki, S. Validity of a dish composition database for estimating protein, sodium and potassium intakes against $24 \mathrm{~h}$ urinary excretion: Comparison with a standard food composition database. Public Health Nutr. 2020, 23, 1297-1306. [CrossRef]

20. Bowman, S.A.; Clemens, J.C.; Thoerig, R.C.; Friday, J.E.; Shimizu, M.; Moshfegh, A.J. Food Patterns Equivalents Database 2011-2012: Methodology and User Guide; Food Surveys Research Group, Beltsville Human Nutrition Research Center, Agricultural Research Service, U.S. Department of Agriculture: Beltsville, MD, USA. Available online: http://www.ars.usda.gov/ba/bhnrc/fsrg (accessed on 20 May 2020).

21. Krebs-Smith, S.M.; Pannucci, T.R.E.; Subar, A.F.; Kirkpatrick, S.I.; Lerman, J.L.; Tooze, J.A.; Wilson, M.M.; Reedy, J. Update of the Healthy Eating Index: HEI-2015. J. Acad. Nutr. Diet. 2018, 118, 1591-1602. [CrossRef]

22. Reedy, J.; Lerman, J.L.; Krebs-Smith, S.M.; Kirkpatrick, S.I.; Pannucci, T.R.E.; Wilson, M.M.; Subar, A.F.; Kahle, L.L.; Tooze, J.A. Evaluation of the Healthy Eating Index-2015. J. Acad. Nutr. Diet. 2018, 118, 1622-1633. [CrossRef]

23. Fulgoni, V.L.; Keast, D.R.; Drewnowski, A. Development and validation of the Nutrient-Rich Foods Index: a tool to measure nutritional quality of foods. J. Nutr. 2009, 139, 1549-1554. [CrossRef] [PubMed]

24. Drewnowski, A. The nutrient rich foods index helps to identify healthy, affordable foods. Am. J. Clin. Nutr. 2010, 91, 1095-1101. [CrossRef] [PubMed]

25. Drewnowski, A.; Rehm, C.D.; Vieux, F. Breakfast in the United States: Food and nutrient intakes in relation to diet quality in national health and examination survey 2011-2014. a study from the international breakfast research initiative. Nutrients 2018, 10, 1200. [CrossRef] [PubMed]

26. Murakami, K.; Shinozaki, N.; Livingstone, M.B.E.; Fujiwara, A.; Asakura, K.; Masayasu, S.; Sasaki, S. Characterization of breakfast, lunch, dinner, and snacks in the Japanese context: An exploratory cross-sectional analysis. Public Health Nutr. 2020, 1-31. [CrossRef]

27. Kroeger, E.N.; Fernandez, J.; Jones, P.; Bertrand, B. Diet quality in early care and education centers: A comparison of menu, served, and consumed lunch measures. J. Nutr. Educ. Behav. 2020, 52, 39-44. [CrossRef]

28. Joyce, J.M.; Rosenkranz, R.R.; Rosenkranz, S.K. Evaluation of variability in dietary quality of school lunches meeting national school lunch program guidelines by socioeconomic status and rurality. Int. J. Environ. Res. Public Health 2020, 17, 8012. [CrossRef]

29. Patel, K.J.; Strait, K.M.; Hildebrand, D.A.; Amaya, L.L.; Joyce, J.M. Variability in dietary quality of elementary school lunch menus with changes in National School Lunch Program Nutrition Standards. Curr. Dev. Nutr. 2020, 4, 1-10. [CrossRef]

30. Murakami, K.; Livingstone, M.B.; Fujiwara, A.; Sasaki, S. Application of the Healthy Eating Index-2015 and the Nutrient-Rich Food Index 9.3 for assessing overall diet quality in the Japanese context: Different nutritional concerns from the US. PLoS ONE 2020, 15. [CrossRef]

31. U.S. Department of Health and Human Services and U.S. Department of Agriculture. 2015-2020 Dietary Guidelines for Americans. 8th Edition. 2015. Available online: https:/ / health.gov/our-work/food-nutrition/2015-2020-dietary-guidelines/guidelines/ (accessed on 21 May 2020).

32. Ministry of Health, Labour and Welfare, Japan. Dietary Reference Intakes for Japanese. 2020. Available online: https://www. mhlw.go.jp/content/10904750/000586553.pdf (accessed on 23 May 2020).

33. Fujiwara, A.; Murakami, K.; Asakura, K.; Uechi, K.; Sugimoto, M.; Wang, H.C.; Masayasu, S.; Sasaki, S. Estimation of starch and sugar intake in a Japanese population based on a newly developed food composition database. Nutrients 2018, 10, 1474. [CrossRef]

34. World Health Organization. Guideline: Sugars Intake for Adults and Children. 2015. Available online: https://apps.who.int/iris/ bitstream/handle/10665/149782/9789241549028_eng.pdf;jsessionid=5AE0B7741981D56DB45F91BACA2F021A?sequence=1 (accessed on 2 June 2020).

35. Greenwood, D.C.; Cade, J.E.; Draper, A.; Barrett, J.H.; Calvert, C.; Greenhalgh, A. Seven unique food consumption patterns identified among women in the UK Women's Cohort Study. Eur. J. Clin. Nutr. 2000, 54, 314-320. [CrossRef]

36. Mangano, K.; Sahni, S.; Kiel, D.; Tucker, K.; Dufour, A.; Hannan, M. Bone mineral density and protein derived food clusters from the Framingham Offspring Study. J. Acad. Nutr Diet. 2015, 115, 1605-1613. [CrossRef] [PubMed]

37. Chen, H.; Ward, M.H.; Graubard, B.I.; Heineman, E.F.; Markin, R.M.; Potischman, N.A.; Russell, R.M.; Weisenburger, D.D.; Tucker, K.L. Dietary patterns and adenocarcinoma of the esophagus and distal stomach. Am. J. Clin. Nutr. 2002, 75, 137-144. [CrossRef] [PubMed]

38. Wirfält, E.; Midthune, D.; Reedy, J.; Mitrou, P.; Flood, A.; Subar, A.F.; Leitzmann, M.; Mouw, T.; Hollenbeck, A.R.; Schatzkin, A.; et al. Associations between food patterns defined by cluster analysis and colorectal cancer incidence in the NIH-AARP diet and health study. Eur. J. Clin. Nutr. 2009, 63, 707-717. [CrossRef] [PubMed]

39. Moeller, S.M.; Reedy, J.; Millen, A.E.; Dixon, L.B.; Newby, P.K.; Tucker, K.L.; Krebs-Smith, S.M.; Guenther, P.M. Dietary patterns: Challenges and opportunities in dietary patterns research. J. Am. Diet. Assoc. 2007, 107, 1233-1239. [CrossRef] [PubMed]

40. Anderson, A.L.; Harris, T.B.; Tylavsky, F.A.; Perry, S.E.; Houston, D.K.; Hue, T.F.; Strotmeyer, E.S.; Sahyoun, N.R. Dietary patterns and survival of older adults. J. Am. Diet. Assoc. 2011, 111, 84-91. [CrossRef]

41. Katagiri, R.; Asakura, K.; Uechi, K.; Masayasu, S.; Sasaki, S. Adequacy of iodine intake in three different Japanese adult dietary patterns: a nationwide study. Nutr. J. 2015, 14, 129. [CrossRef] 
42. Poti, J.M.; Duffey, K.J.; Popkin, B.M. The association of fast food consumption with poor dietary outcomes and obesity among children: Is it the fast food or the remainder of the diet? Am. J. Clin. Nutr. 2014, 99, 162-171. [CrossRef]

43. Kim, J.; Yu, A.; Choi, B.Y.; Nam, J.H.; Kim, M.K.; Oh, D.H.; Yang, Y.J. Dietary patterns derived by cluster analysis are associated with cognitive function among Korean older adults. Nutrients 2015, 7, 4154-4169. [CrossRef]

44. Murakami, K.; Shinozaki, N.; Fujiwara, A.; Yuan, X.; Hashimoto, A.; Fujihashi, H.; Wang, H.C.; Livingstone, M.B.E.; Sasaki, S. A systematic review of principal component analysis-derived dietary patterns in Japanese adults: Are major dietary patterns reproducible within a country? Adv. Nutr. 2019, 10, 237-249. [CrossRef]

45. Suga, H.; Asakura, K.; Sasaki, S.; Nojima, M.; Okubo, H.; Hirota, N.; Notsu, A.; Fukui, M.; Date, C. Effect of seasonality on the estimated mean value of nutrients and ranking ability of a self-administered diet history questionnaire. Nutr. J. 2014, 13, 51. [CrossRef]

46. Subar, A.F.; Freedman, L.S.; Tooze, J.A.; Kirkpatrick, S.I.; Boushey, C.; Neuhouser, M.L.; Thompson, F.E.; Potischman, N.; Guenther, P.M.; Tarasuk, V.; et al. Addressing current criticism regarding the value of self-report dietary data. J. Nutr. 2015, 145, 2639-2645. [CrossRef] [PubMed]

47. Livingstone, M.B.E.; Black, A.E. Markers of the validity of reported energy intake. J. Nutr. 2003, 133, 895S-920S. [CrossRef] [PubMed]

48. Lo Siou, G.; Yasui, Y.; Csizmadi, I.; McGregor, S.E.; Robson, P.J. Exploring statistical approaches to diminish subjectivity of cluster analysis to derive dietary patterns. Am. J. Epidemiol. 2011, 173, 956-967. [CrossRef] [PubMed] 